\title{
Red Algal Extremophiles: Novel Genes and Paradigms
}

\section{Julia Van Etten}

Graduate Program in Ecology and Evolution, Rutgers University, New Brunswick, NJ 08901

julia.vanetten@rutgers.edu

\begin{abstract}
The Cyanidiophyceae are a class of unicellular red algae that live in environments at the extremes of temperature, $\mathrm{pH}$, salt, and heavy metal concentrations. These photosynthetic organisms have been able to occupy these rather unusual niches by acquiring a variety of genes from extremophilic prokaryotes and/or viruses via the process of horizontal gene transfer (HGT). Elucidating how the proteins encoded by these genes integrate into existing metabolic systems is crucial to understanding and evaluating the significance of HGT in eukaryotes and its role in conferring adaptive traits. This article addresses the fundamentals of HGT in model red algae, its function as a driver of evolution across the web of life with a focus on eukaryotes, and summarizes ongoing work and the future directions of this field.
\end{abstract}

Keywords: red algae, eukaryotes, horizontal gene transfer, adaptation, extremophiles

\section{Introduction}

Successful adaptation to a new or changing environment is the hallmark of evolutionary success for any organism. Whether that means corals ultimately adapting to prolonged heat stress due to climate change, plants occupying new urban niches, or changes to the human population following a global pandemic, stressful environmental conditions drive evolution by selecting for traits encoded by the genes that make up genomes. Most organisms cannot cope with every new stressor; after all, $99.9 \%$ of species that have ever lived are now extinct [1]. So how would a group of red algae that were previously occupying mesophilic habitats (moderate, such as oceans and lakes) evolve to thrive in some of the most extreme and inhospitable environments on Earth?

The Cyanidiophyceae (example pictured in Figure 1) are unicellular red algae found in aquatic habitats worldwide. What makes them different from other algae and most other organisms on the planet is that some species are able to survive and reproduce under a myriad of extreme environmental conditions. These include high temperatures up to $56^{\circ} \mathrm{C}$ $\left(\sim 133^{\circ} \mathrm{F}\right)$, high and low light, $\mathrm{pH}$ that approaches 0 , and high concentrations of salt and toxic chemicals like mercury and arsenic [2]. This combination of factors allows these organisms to qualify as extremophiles, and because they live in many different kinds of extremes, they are actually termed "polyextremophiles." It is important to note most other red algae (Rhodophytes) live in mesophilic conditions and that the common ancestor of this clade was likely a mesophile as well [3] (Figure 2). This means that the polyextremophilic members of the Cyanidiophyceae acquired the ability to thrive under extreme conditions at some point in their evolutionary history, that is, they were not ancestral traits of all red algae. Because this lifestyle strategy was advantageous to their survival, it became fixed within populations, allowing them to be the only photosynthetic organelle (plastid)-bearing organisms to live in such volatile conditions (Figure 3). But how is this possible? And why were these algae able to adapt to extremes while others have not? Both genomic and microscopy techniques are important tools in analyzing genetic diversity and the adaptations organisms have made to living in extreme environments.

\section{Horizontal Gene Transfer}

The first important concept to keep in mind is the distinction between prokaryotes and eukaryotes. Prokaryotes are tiny, single-celled or filamentous organisms that lack a nucleus (Figure 4). They have rigid cell walls that protect them from the outside world, and they obtain their energy from light, by breaking down inorganic molecules in their environment, or by consuming organic molecules necessary for survival. Prokaryotes include all bacteria and archaea on Earth. These organisms reproduce asexually by splitting into two identical cells, a process called binary fission. They can also exchange genes between one another in a process called conjugation, where they swap small rings of DNA (called plasmids) with each other, or they can receive new bits of genetic information from viruses (transduction), or from the external environment (transformation). When they reproduce asexually, they pass their genes on to the next generation, from parent to offspring, and their offspring can then reproduce and continue this pattern. This process is vertical (Figure 5a). However, when they receive foreign genetic information during their lifetime, they can ultimately pass on these new genes not present in their ancestors. This process is called horizontal gene transfer (HGT) (Figure 5b) and is the most common way prokaryotes add genetic diversity and new, often adaptive traits to their populations [4].

In contrast, eukaryotic cells are quite different. Although some are single-celled or filamentous like most protists (including algae), many, like most plants, fungi, and animals, are multicellular. What distinguishes eukaryotes from prokaryotes is that each of their cells contains a nucleus where most or all of their DNA is enclosed by a membrane (Figure 4). This, and the fact that DNA transcription occurs in the protected nucleus, whereas the decoding of mRNA to create proteins occurs in the cytoplasm, makes it more difficult for eukaryotes to receive genetic material from external sources. They also all (or nearly all) have membrane-bound organelles like mitochondria, which allow them to take in organic molecules and convert them to energy. Some eukaryotes, like plants and algae, also have plastids, which allow them to convert light energy into chemical energy via photosynthesis. Mitochondria and plastids were once free-living prokaryotes billions of years ago before they were taken in by a host eukaryote and permanently maintained via the processes of endosymbiosis and organellogenesis. These features are some 


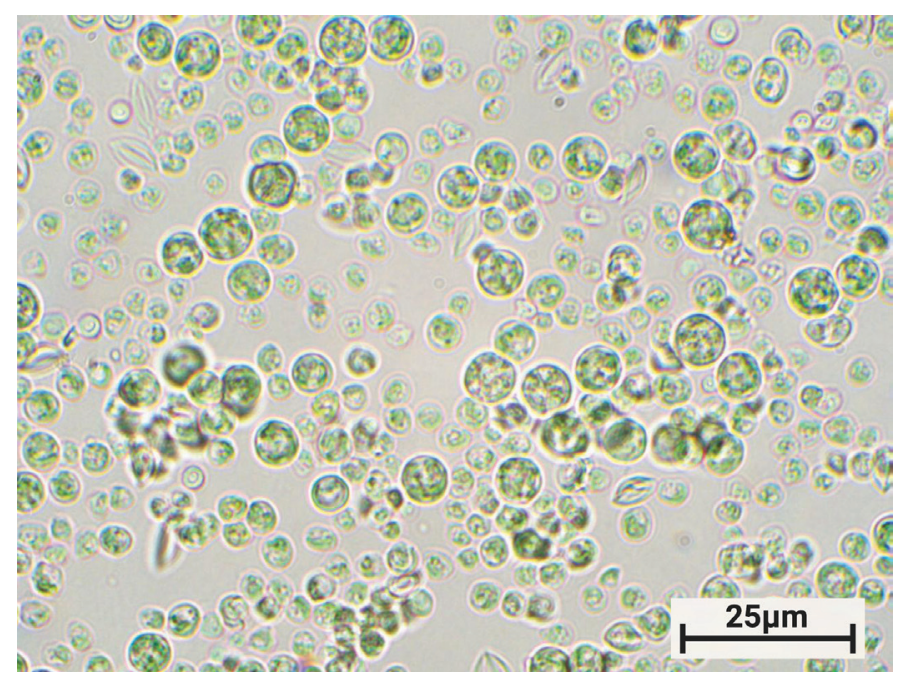

Figure 1: Photomicrograph of Galdieria sulphuraria strain YNP5572. Pictured at $1000 \times$ total magnification (TM). Brightfield. Their color warrants an explanation: members of the "red" algae, the Cyanidiophyceae are actually green. Before more modern methods of sequencing and classification, this made researchers think that they were other types of algae, like green or glaucophyte. They actually got their green color because, as they moved to higher light environments, they evolved to no longer need the red pigment phycoerythrin, and thus they no longer reflect red light [40].

of the characteristics that make the cells of eukaryotes more complex than prokaryotes. Moreover, there are both asexually and sexually reproducing eukaryotes; both strategies are responsible for transferring genetic information to the next generation vertically (Figure 6a).

Traditionally, eukaryotes were thought to rely heavily on the processes of gene duplication and mutation that allow traits to be selected for by the forces of evolution. Novel adaptation of these traits allowed organisms to evolve [5]. These are slow processes and because of this, eukaryotes are not able to acquire new genes as quickly as prokaryotes. Based on this paradigm, it would be an extremely lengthy and unlikely process for an organism to acquire the genetic properties necessary to go

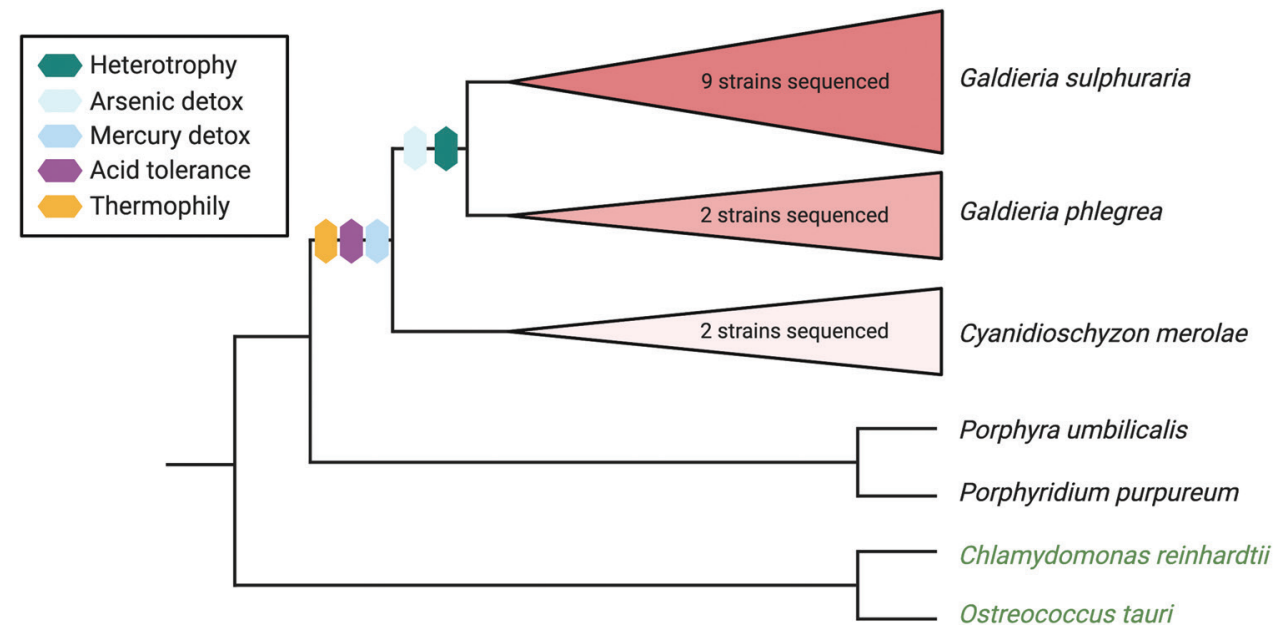

Figure 2: Schematic bifurcating phylogenetic tree showing three Cyanidiophyceaen red algae species and some of the extremophilic traits they have acquired. Topology based on tree from Rossoni et al. 2019 [12]. Outgroup taxa include two other red algae ( $P$. umbilicalis, $P$. purpureum) and two green algae (C. reinhardtii, O. tauri). from a regular aquatic environment to one where it would need genes for temperature tolerance, salt tolerance, acid tolerance, light fluctuations, and xenobiotic/heavy metal detoxification, as in the case of the Cyanidiophyceae. However, as the field of genomics has progressed and more work studying the development of novel genes has been published, it is now known that gene and genome duplications are only two of many diverse pathways that lead to the development of novel genes [6]. One such pathway that is now the topic of many studies in the mainstream scientific literature is HGT. Because eukaryotic cells are more complex than prokaryotic cells, it was previously thought that they generally could not acquire new genes horizontally like bacteria, and when they did it was extremely uncommon and mostly in the form of endosymbiotic gene transfer (discussed below) [7]. However, over the past twenty years or so, genome sequencing projects have led to an accumulation of examples of HGT in eukaryotes [8], including work from our collaborators and in our lab that has suggested that these HGTs confer novel adaptive traits to their recipient genomes [9-12]. Just like in prokaryotes, HGT is proving to be an important fundamental mechanism of evolution, and especially in microbial eukaryotes like the Cyanidiophyceae that can more easily pass on HGTs due to their unicellular nature. That is, each cell is a germ cell and can propagate novel HGTs in the population via mitosis.

There are a variety of mechanisms whereby a eukaryote can acquire new genes horizontally (Figure 6b). One method is the uptake of exogenous genetic material from the environment (analogous to transformation in prokaryotes; Figure $6 \mathrm{~b}(\mathrm{i})$ ), whereby bits of nucleic acids with properties that allow them to insert themselves into the nuclear DNA of a eukaryotic cell, are taken up and incorporated. Another is via phagocytosis, the process by which one cell or unicellular organism engulfs a substance (which can be another cell/organism) and brings it into the cell where it is typically digested. It is possible for gene transfer to occur during this process where genetic material is transferred from the consumed cell/organism to the nucleus of the phagocytic cell [13] (Figure 6b(ii)). A third method is for a eukaryotic cell to be infected by a virus that injects DNA or RNA into the host cell in order to make more viruses, and during this process some of the viral genetic material gets incorporated into the host genome (Figure 6b(iii)). Another method, similar to exogenous uptake, is when some eukaryotic organisms (for example, some red algae; Porphyridium purpureum $[14,15]$ ) actually have plasmids of their own and can host bacterial plasmids in their nucleus where they can replicate and likely incorporate plasmid DNA into other DNA-bearing entities like organelles, the red algal nuclear DNA, or genomes of other organisms (Figure $6 \mathrm{~b}(\mathrm{iv}))$. Lastly, when a cell ingests another cell but does not digest it, it can become an endosymbiont and, a 

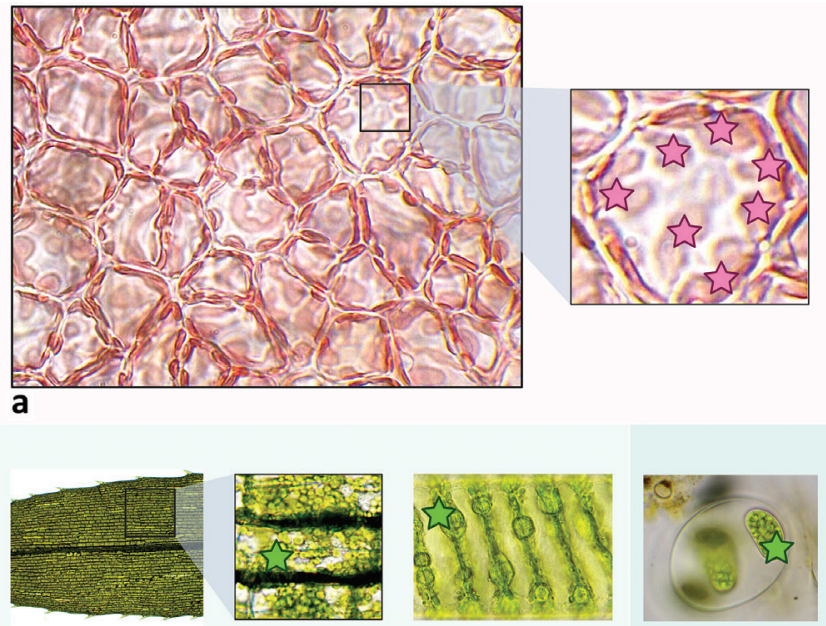

b

Figure 3: Examples of plastids across Archaeplastida lineages. Plastids in each example are denoted by stars. a. Surface view of the cortex of red alga Agardhiella sp. viewed at $1000 \times$ TM and then magnified further digitally after initial micrography. Here, the plastids are visible as elongated and amorphous light red spots along the outer borders of the cells. b. Micrographs showing plastids in other major Archaeplastida groups. From left to right: leaf of Elodea $\mathrm{sp}$. (an aquatic tracheophyte) magnified to $400 \times$ TM showing many plastids within each cell; view of a single cell of Spirogyra sp., a filamentous green alga (charophyte) at $1000 \times$ TM showing a spiral arrangement of chloroplasts; a small colony of glaucophyte algae (ID unconfirmed but possibly a Glaucocystis sp.) at $400 \times$ TM with cells containing plastids ("cyanelles").
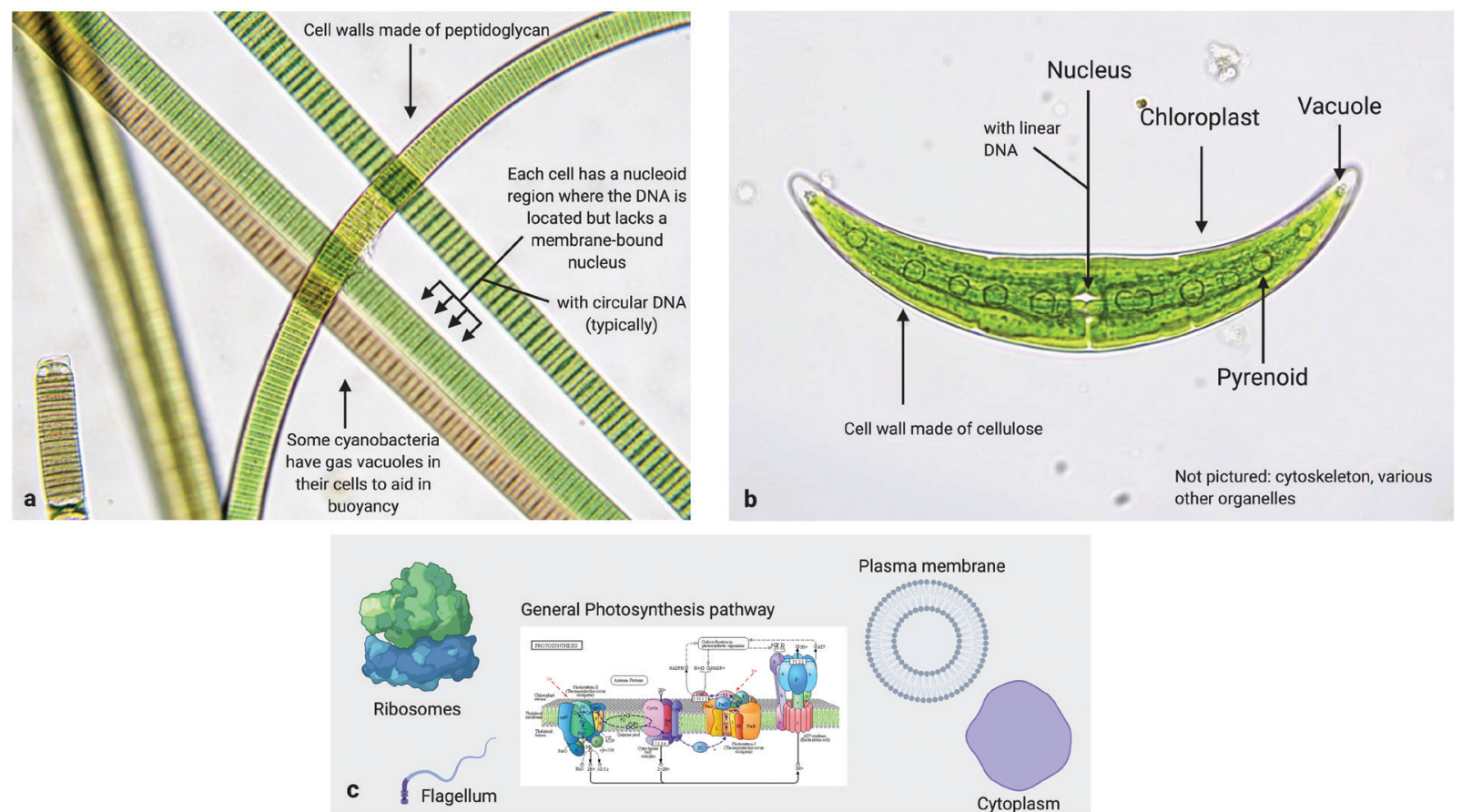

General Photosynthesis pathway
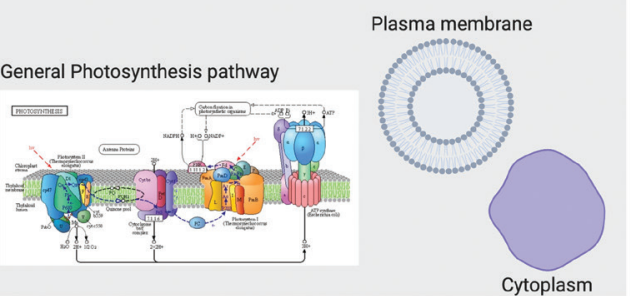

few times in evolutionary history, endosymbionts have become bona fide energy-producing organelles like mitochondria and plastids [see 7,16,17]. Once these cells become fixtures within the host cell, they undergo massive genome reduction, and many of their genes are transferred to the host nucleus in a process called endosymbiotic gene transfer (EGT). In order to make sure that despite losing many genes, this cell-turnedorganelle can still function in a useful way, the host nucleus must contain an adequate repertoire of genes to complement the organelle's remaining functions $[18,19]$ (Figure $6 \mathrm{~b}(\mathrm{v}))$. Furthermore, because certain pathways must be conserved for the organelle to remain functional, this might not be possible between the organellar genome and the host genome alone following such massive genome reduction and EGT. To remedy this problem, it has been shown that genes contemporaneously acquired horizontally from other sources can compensate for missing genes from the other two sources. It is hypothesized that plastids would not be what they are currently without gene complementation via HGTs from Chlamydial cells that were incorporated into the ancestral plastid-bearing organism's nuclear DNA [20].

\section{Cyanidiophyceae as Models for Studying HGT}

It is by HGT that Cyanidioschyzon spp. and Galdieria spp. (Figure 1), the two genera of Cyanidiophyceae that have been sequenced and analyzed, have acquired new genes with novel functions, likely from many different extremophile 
a)

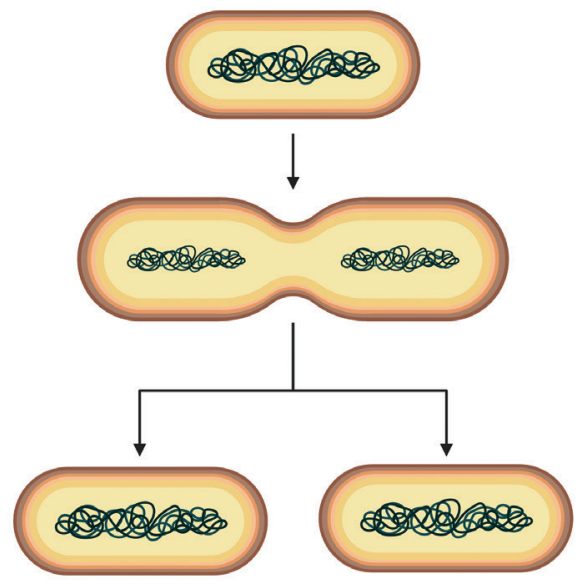

b)

Horizontal

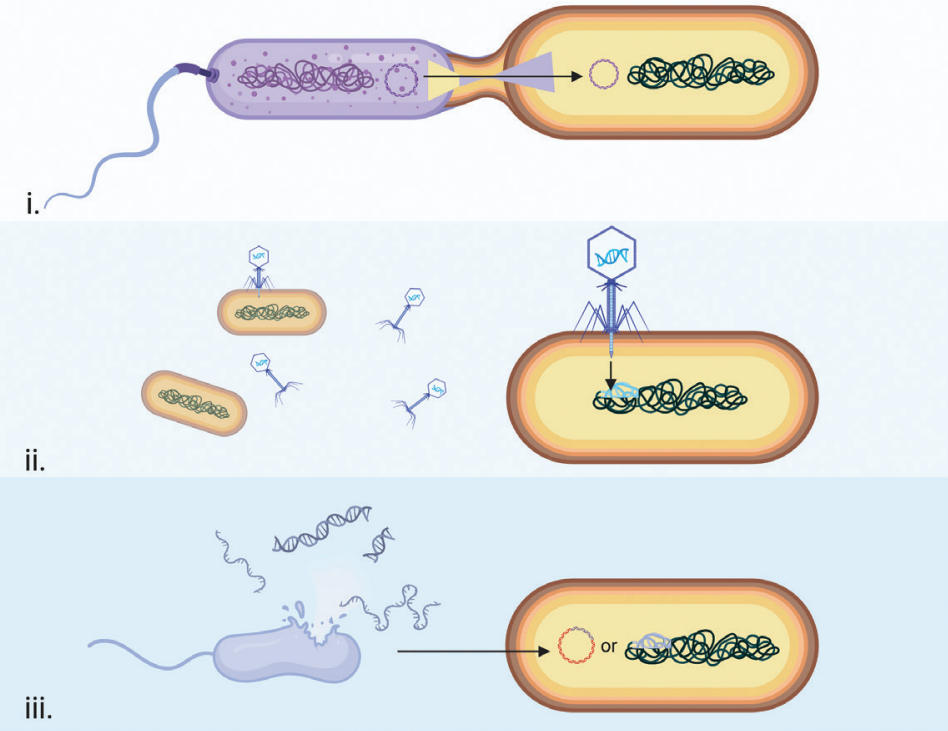

Figure 5: Gene transfer mechanisms in prokaryotes. (a.) A schematic depiction of generic prokaryote asexual reproduction via binary fission, the primary form of vertical transfer of genes. (b.) General mechanisms of prokaryote horizontal gene transfer. i. Conjugation; ii. Transduction; iii. Transformation.

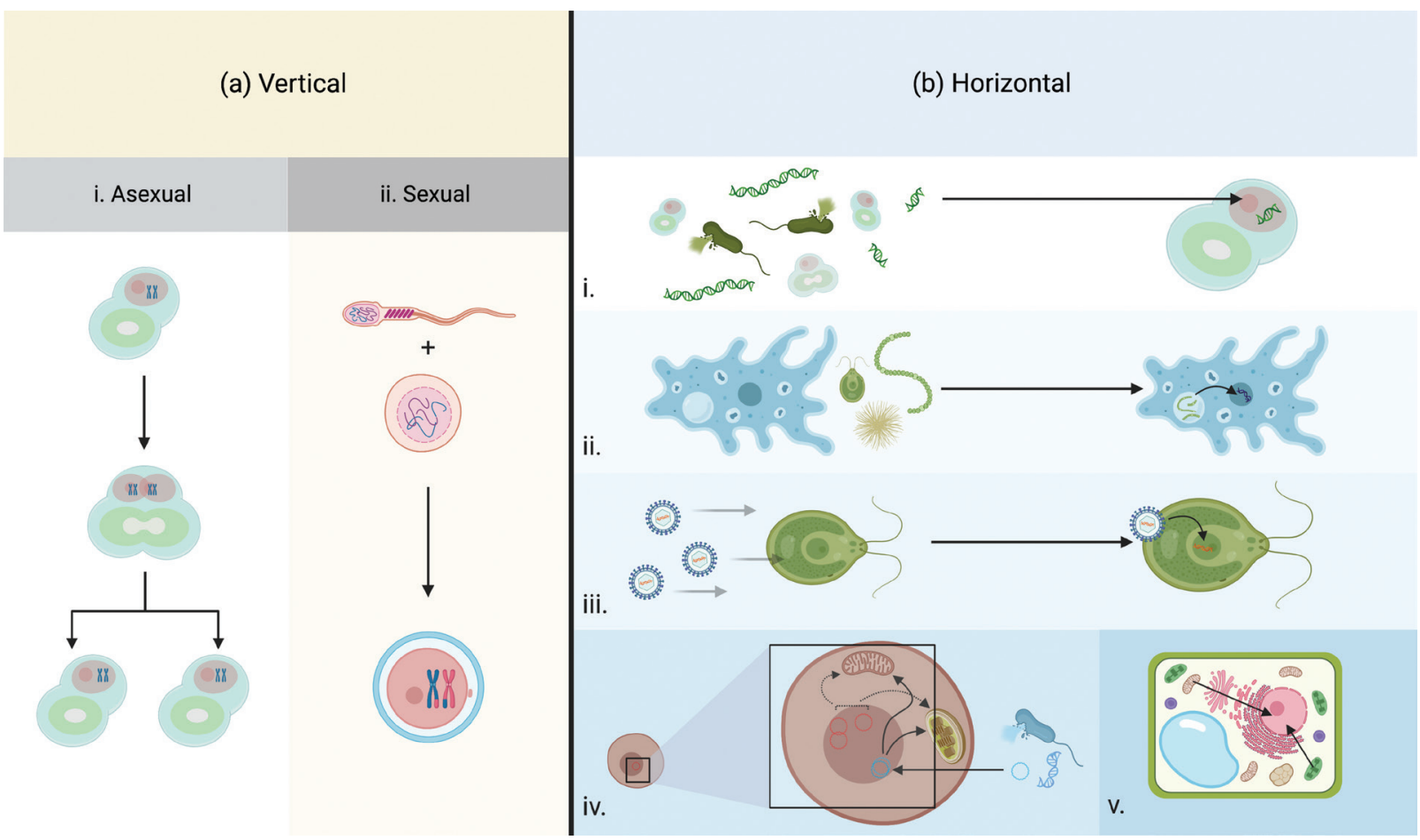

Figure 6: Gene transfer mechanisms in eukaryotes. (a.) Vertical mechanisms. i. A schematic depiction of asexual reproduction in eukaryotes (cartoon is of a hypothetical Cyanidioschyzon-like unicellular red alga) via binary fission; ii. Schematic of generic sexual reproduction in a hypothetical animal. (b.) Horizontal mechanisms. i. Uptake of exogenous DNA: into a hypothetical Cyanidioschyzon-like alga (Cyanidioschyzon sp. do not have cell walls so it is easier to take in external molecules); ii. Phagocytosis: a hypothetical amoeba ingests a variety of other organisms (left), including cyanobacteria which are pictured inside a food vacuole (right), and DNA is subsequently transferred to the nucleus during digestion; iii. Viral infection: of a hypothetical Chlamydomonas-like green alga; iv. Plasmid-related activity in a hypothetical Porphyridium-like red alga with algae plasmids transferring genes (red) and bacterial plasmids replicating in the algal nucleus and subsequently transferring genes (blue); v. Endosymbiotic gene transfer: genes from the chloroplasts and mitochondria in this plant cell are transferred to the plant cell nucleus. 
prokaryote donors that live in the same environment, or from viruses. Previous work in our lab (the Bhattacharya lab at Rutgers University) and with our collaborators has confirmed this bioinformatically through orthogroup analysis and phylogenomic methods $[11,12]$, showing that these putative HGTs found across various species and strains of these algae are more similar to genes found in prokaryotes than in the algal web of life. My current work, funded by a NASA FINESST grant in Planetary Science, is to validate these findings functionally, by testing what these genes do in the extremophile Cyanidiophyceae and how their encoded functions have been incorporated into existing metabolic networks.

Most genes that are horizontally transferred to a new genome are quickly lost. These are transient "failed experiments" of evolution that we will never know about. In order to become a universal feature of the species (fixed), they must outlast the other fundamental forces of evolution like genetic drift and natural selection that would lead to the removal of the new gene from the population. One way for an HGT-derived protein to overcome these obstacles is for it to occupy a position in a pre-existing metabolic pathway that allows it to be functionally integrated into the new genome. Once there, if its presence increases fitness of the organism, the foreign gene may subsequently undergo duplications that allow it to enhance its functional benefit even further. There are examples of this in many eukaryotic organisms including the green alga Ulva mutabilis, the gastrointestinal parasite Blastocystis sp., and beetles [21,22,23]. Our group has already shown that such HGTs exist, making up approximately $1 \%$ of cyanidiophyceaen genomes, and that they appear to provide adaptive advantages by conferring extremophilic traits to these organisms, which act as drivers of evolution, and allow them to occupy such hostile niches. The next step, which is currently underway, is to test this hypothesis by doing experiments to measure expression levels of genes (including HGTs) across a variety of physical and chemical conditions and then modeling the dynamics of the metabolic networks that the HGT-derived proteins have become a part of to show how they function in congruence with native proteins.

Some of the HGTs acquired by members of this lineage make these algae rather unusual. For example, algae are photosynthetic; that is, they have a plastid and obtain energy primarily from light. But interestingly, all strains of Galdieria that we grow in our lab can live in complete darkness, which doesn't seem to make sense! That would be like putting a houseplant in a totally dark room indefinitely and expecting it to grow. Galdieria can do this because the ancestor of this lineage had acquired genes for heterotrophy (consuming food, not making its own) that allowed the organism to feed on carbon sources like glucose and glycerol when light is not available [12]. Sugar uptake must have become advantageous in the population that acquired the genes for it; the algal cells that could take up fixed carbon could also produce energy in both the presence and absence of light compared to other cells that could only generate energy when light was available. This increased the fitness of the heterotrophic cells, which would have led to selection of these individuals and, ultimately, for the HGTs that conferred this trait to reach fixation in the Galdieria population.

\section{Algae on Earth and Algae in Space?}

Aside from their importance to our ongoing research, the Cyanidiophyceae are fascinating study systems for the insights they provide to fields like ecology and astrobiology. Rhodophyta (the red algal clade) is among the earliest branching lineages (it diverged ca. 1.4 Ga) of organisms in Archaeplastida (the superphylum that contains all primary plastid-bearing organisms except for Paulinella sp., that is, algae and land plants), and the Cyanidiophyceae represent the first major split within Rhodophyta (Figure 7). Studying such organisms provides insights into ancient traits and physiologies. As primary producers, algae ("algae" here refers to all "phytoplankton" which include organisms with secondary and tertiary plastids) are important ecologically, and along with Cyanobacteria, they produce nearly one-half of the oxygen present in the atmosphere (land plants produce the other $\sim 54 \%$ ) [24] and serve as the food source for organisms at higher trophic levels. Atmospheric oxygen likely played a causative role in the evolution of multicellular tissuebearing organisms like Metazoans (animals) whose radiation has contributed to the vast biodiversity on Earth today [25]. Furthermore, large blooms of algae can lead to anoxia in aquatic environments and result in cascading effects to food webs that kill many organisms and cause economic and sometimes medical harm to humans [26]. Algae also have important applications in bioremediation and biofuel production, therefore, understanding their evolution and physiology has broad impacts on many human endeavors that are often overlooked by non-phycologists [27].

The ability to convert arsenic and mercury to less toxic forms make Galdieria and Cyanidioschyzon good bioremediators, and this allows them to survive in geothermal environments with very high concentrations of these elements. Some well-studied examples of such locations are the hot springs of Yellowstone National Park and Soos National Park in the Czech Republic. Not only do these habitats provide the kinds of stresses that facilitated the transition to polyextremophily in these algae and provide a niche where these organisms can thrive without a lot of competition, they also resemble conditions of the early Earth as well as the volatile conditions that may exist on exoplanets elsewhere in the universe. Currently lacking the capability to explore such locales, it helps to be able to study organisms on Earth that are thriving in such conditions and to use these habitats as a proxy for how life has thrived in the past and may live elsewhere. This allows us, along with our collaborators at NASA, to gain a firsthand understanding of some of the biochemical and molecular processes associated with the biological limits of life as we know it. Although it would be astronomically unlikely for algae to be found somewhere else in the universe, it is less unlikely that some sort of nucleic acid-based lifeform would exist [28]. With nucleic acids like DNA and RNA come proteins, and proteins form the basis of metabolic networks. Understanding how genes are exchanged between organisms and how novel genes arise and trigger rapid adaptation adds to our fundamental knowledge of genomics and evolution, as well as the environmental limits of life that we can study firsthand on Earth. This kind of work will inform the search for habitable worlds elsewhere and the study of extraterrestrial life if it is found in the future. 


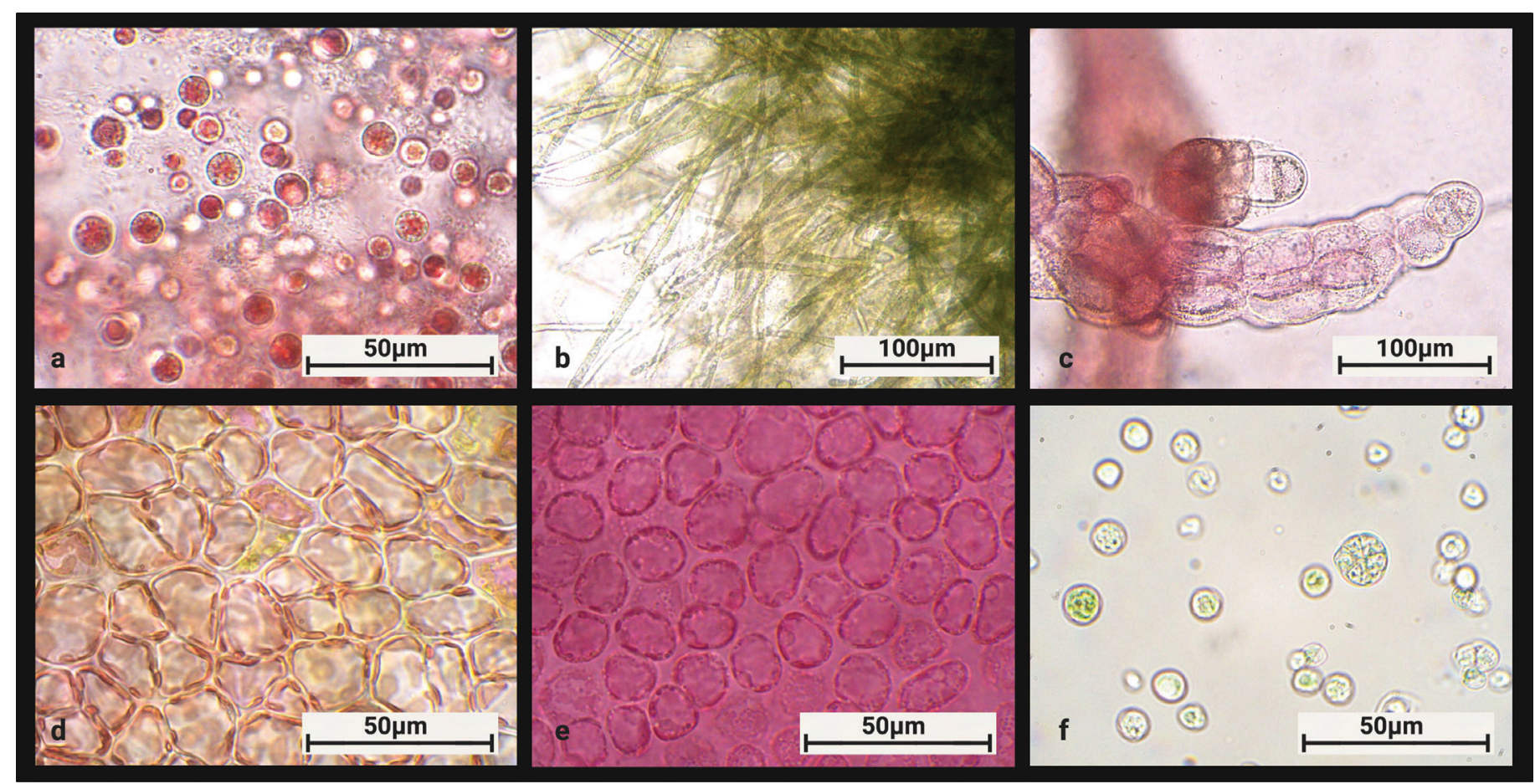

Figure 7: Composite of six red algae genera representing many major groups within the Rhodyphyta. (a.) Porphyridium sp. (b.) Batrachospermum sp. (c.) Polysiphonia sp. (d.) Agardhiella sp. (outer cortex view) (e.) Rhodymenia sp. (f.) Galdieria sulphuraria.

\section{Learning More about HGT in Eukaryotes}

Although the Cyanidiophyceae have HGTs as approximately $1 \%$ of their gene inventory and this makes them good models for studying this process, they are certainly not unique. Many other extremophilic eukaryotes have HGTs, ranging from protists like ciliates and dinoflagellates [29,30] to desiccation and radiation-tolerant animals like rotifers [31]. One example is ice-binding proteins in Arctic/Antarctic diatoms that were acquired from prokaryotes or viruses that survive cold temperatures, giving diatoms the ability to more easily adapt to polar climates [32]. When considering extremophiles, traits related to extremophily are rather conspicuous, allowing us to more easily identify the impact of more noticeable HGT events. However, studies in recent years have shown that HGTs are in fact ubiquitous across the eukaryotic tree of life and that they also play equally important roles in lifestyle changes not related to extremophily. One example is mesophilic diatoms that have acquired genes for ferritin, a protein that allows them to sequester iron, which is typically a limiting nutrient in marine environments [33]. HGTs have also been implicated in the transition of some organisms from free-living to parasitic lifestyles and in the colonization of land by plants $[34,35,36]$. Moreover, HGTs don't always have to be from prokaryotes; there are many cases of eukaryote-to-prokaryote and eukaryote-toeukaryote HGT as well. One example is the oomycetes, a group of protists that live as plant parasites and thus behave similarly to fungal parasites of plants. It turns out that across the oomycete species surveyed, all have acquired a variety of HGTs from fungi, and this has not only allowed them to become more efficient plant pathogens, but they are so convergently similar to their fungal gene donors that they were mistakenly classified as fungi when originally discovered [37,38].

While these examples provide exciting insights, they are not comprehensive enough for us to come to robust universal conclusions. A majority of eukaryotic diversity is still largely unexplored, especially in fungi and protists. As more genomes are sequenced and cryptic and rare species are identified, the eukaryotic tree of life can be resolved with a higher degree of precision. Molecular methods of characterization are one important way to go about this work, but they come with biases that may hinder developing a more exhaustive survey of eukaryotes, which is why it is also important to complement these types of analyses with conventional light microscopy [39], a practice that has dwindled in this field due to time constraints and reliance on molecular methods. Microbial eukaryotes like protists are vastly understudied, under-classified, and overlooked in most textbooks and biology classes. One of the best ways to learn about them will always be to simply observe them. Using these skills to catalogue these microorganisms is important for understanding broad patterns in eukaryotic ecology and genomics, as well as finding new models for interesting processes like HGT integration. Novel species can be identified and collected in the hot springs of Yellowstone National Park like the Cyanidiophyceae once were, or they can be found in a local pond or soil sample. Maybe an unknown alga in a pond has overcome a unique set of desiccation challenges and the resulting adaptations are evident in their genome architecture, or maybe a diatom lineage has begun to diverge after many generations living amongst pollution and a unique assemblage of 


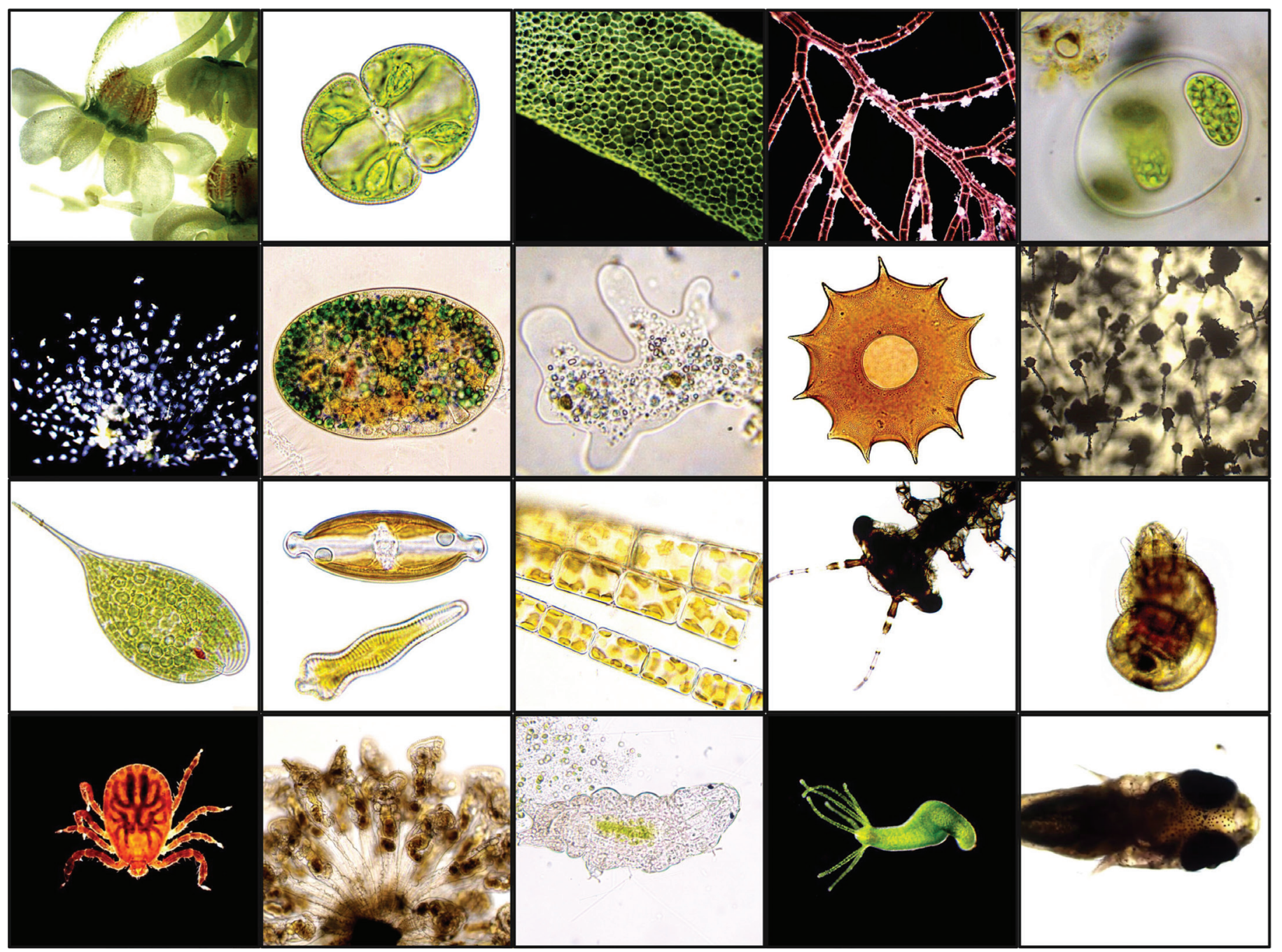

Figure 8: Composite of many microscopic or small eukaryotic organisms giving a snapshot of eukaryotic diversity. All photos were taken by Julia Van Etten as part of her "Couch Microscopy" project (www.instagram.com/couch_microscopy). From left to right and then top to bottom: Daucus carota (wild carrot), Cosmarium sp. (green algae), Hydrodictyon sp. (green algae), Polysiphonia sp. (red algae), Glaucocystis sp. (glaucophyte algae), Carchesium sp. (protozoan), Nassula sp. (protozoan), amoeba (genus unknown), Arcella dentata (testate amoeba), Aspergillus sp. (mold), Phacus sp. (Euglena), diatoms: Navicula sp. (top), Gomphonema sp. (bottom), Melosira sp. (filamentous diatoms), damselfly larva, freshwater snail, longhorned tick (Haemaphysalis longicornis), colonial rotifers (Sinantherina socialis), tardigrade, Hydra viridissima, unidentified juvenile freshwater fish.

microbial cohabitants in an urban body of water. Untapped eukaryote diversity needs to be addressed in order to add biodiverse representation to and eliminate bias from growing fields like the study of eukaryotic HGT and novel gene development. Figure 8 shows a variety of eukaryotes found in New Jersey, many of which have yet to be sequenced or analyzed for HGT.

\section{Concluding Remarks}

The Cyanidiophyceae are a group of red algae that have acquired a useful array of HGTs conferring adaptive traits that allow them to evolve in hostile environments reminiscent of the early Earth and possibly exoplanets. They are unusual in terms of the niches they occupy as polyextremophilic eukaryotes but are not unusual among eukaryotes with respect to their possession of HGTs. As more eukaryote genomes are sequenced and mined for
HGTs, it becomes ever more apparent that these genes play a significant role in eukaryotic evolution. Understanding the dynamics of HGT-derived proteins within native metabolic networks is the next step in elucidating their functional role. In order to more exhaustively understand HGT and its broad impact across the eukaryotic tree of life, it is necessary to sequence and characterize more organisms, particularly cryptic species via both molecular phylogenomic methods as well as microscopy.

\section{Acknowledgements}

JVE's work is supported by NASA Future Investigators in NASA Earth and Space Science and Technology (FINESST grant 80NSSC19K1542). JVE would like to acknowledge Prof. Debashish Bhattacharya and all members of the Bhattacharya lab for their help and support with this research as well as Dr. Lynn Rothschild and the members of the Rothschild lab. 
JVE would also like to thank Kyle T. David (https://orcid. org/0000-0001-9907-789X) and Dylan Simpson (https://orcid. org/0000-0003-1515-868X) for their feedback. Figures 2-8 were created with Biorender.com.

\section{References}

[1] E Mayr, This Is Biology: The Science Of The Living World, Universities Press, 1997, pg. 148.

[2] RW Castenholz and TR McDermott, Red Algae in the Genomic Age, Springer Netherlands, 2010, pp. 357-71.

[3] V Reeb and D Bhattacharya, Red Algae in the Genomic Age, Springer Netherlands, 2010, pp. 409-26.

[4] EV Koonin, F1000Res 5 (2016) 1805.

[5] JS Taylor and J Raes, Annu Rev Genet 38 (2004) 615-43.

[6] M Long et al., Annu Rev Genet 47 (2013) 307-33.

[7] WF Martin, BioEssays 39(12) (2017) 201700115.

[8] J Van Etten and D Bhattacharya, Trends in Genetics (2020) doi:10.1016/j.tig.2020.08.006.

[9] G Schönknecht et al., Science 339 (2013) 1207-10.

[10] H Qiu et al., Current Biology 23 (2013) R865-66.

[11] G Schönknecht et al., BioEssays 36 (2014) 9-20.

[12] AW Rossoni et al., eLife 8 (2019) e45017.

[13] L Holmgren, Biochem Biophys Res Comm 396 (2010) 147-51.

[14] J Lee et al., Sci Rep 6 (2016) 23744-57.

[15] Z Li and R Bock, Nat Commun 9 (2018) 3451-58.

[16] H Imachi et al., Nature 577 (2020) 519-25.

[17] A Gabr et al., J Phycol 56(4) (2020) 837-43.

[18] S Karkar et al., Proc Natl Acad Sci USA 112 (2015) 10208-15.

[19] JF Allen, J Theoretical Biol 434 (2017) 50-57.

[20] A Moustafa et al., PLoS ONE 3 (2008) e2205.

[21] O De Clerck et al., Current Biology 28 (2018) 2921-33.e5.

[22] L Eme et al., Current Biology 27 (2017) 807-20.

[23] R Kirsch et al., Insect Biochem Mol Biol 52 (2014) 33-50.

[24] CB Field et al., Science 281 (1998) 237-40.

[25] DB Mills and DE Canfield, BioEssays 36 (2014) 1145-55.

[26] LM Grattan et al., Harmful Algae 57 (Pt.B) (2016) 2-8.

[27] SK Mandotra et al., Restoration of Wetland Ecosystem: A Trajectory Towards a Sustainable Environment, Springer Singapore, 2020, pp. 145-60.

[28] TM McCollom, Annu Rev Earth Planet Sci 41 (2013) 207-29.

[29] S Pucciarelli et al., Antarctic Sci 26 (2014) 491-501.

[30] TG Stephens et al., BMC Biology 18(1) (2020) 56-76.

[31] Y Yoshida et al., Horizontal Gene Transfer, Springer, 2019, pp. 203-26.

[32] JA Raymond and HJ Kim, PLoS ONE 7(5) (2012) e35968.

[33] A Marchetti et al., Nature 457 (2009) 467-70.

[34] J Xiong et al., Sci Rep 5 (2015) 15470-81.

[35] S Cheng et al., Cell 179 (2019) 1057-67.e14.

[36] S Wang et al., Nat Plants 6 (2020) 95-106.

[37] TA Richards et al., PNAS 108 (2011) 15258-63.

[38] JO Andersson, Current biology 16(18)(2006) R804-06.

[39] PJ Keeling and F Burki, Current Biology 29 (2019) R808-17.

[40] S Lin et al., Plant Physiol 93 (1990) 772-77.

[41] M Kanehisa, Methods Mol Biol: Protein Function Prediction 1611 (2017) 135-45.

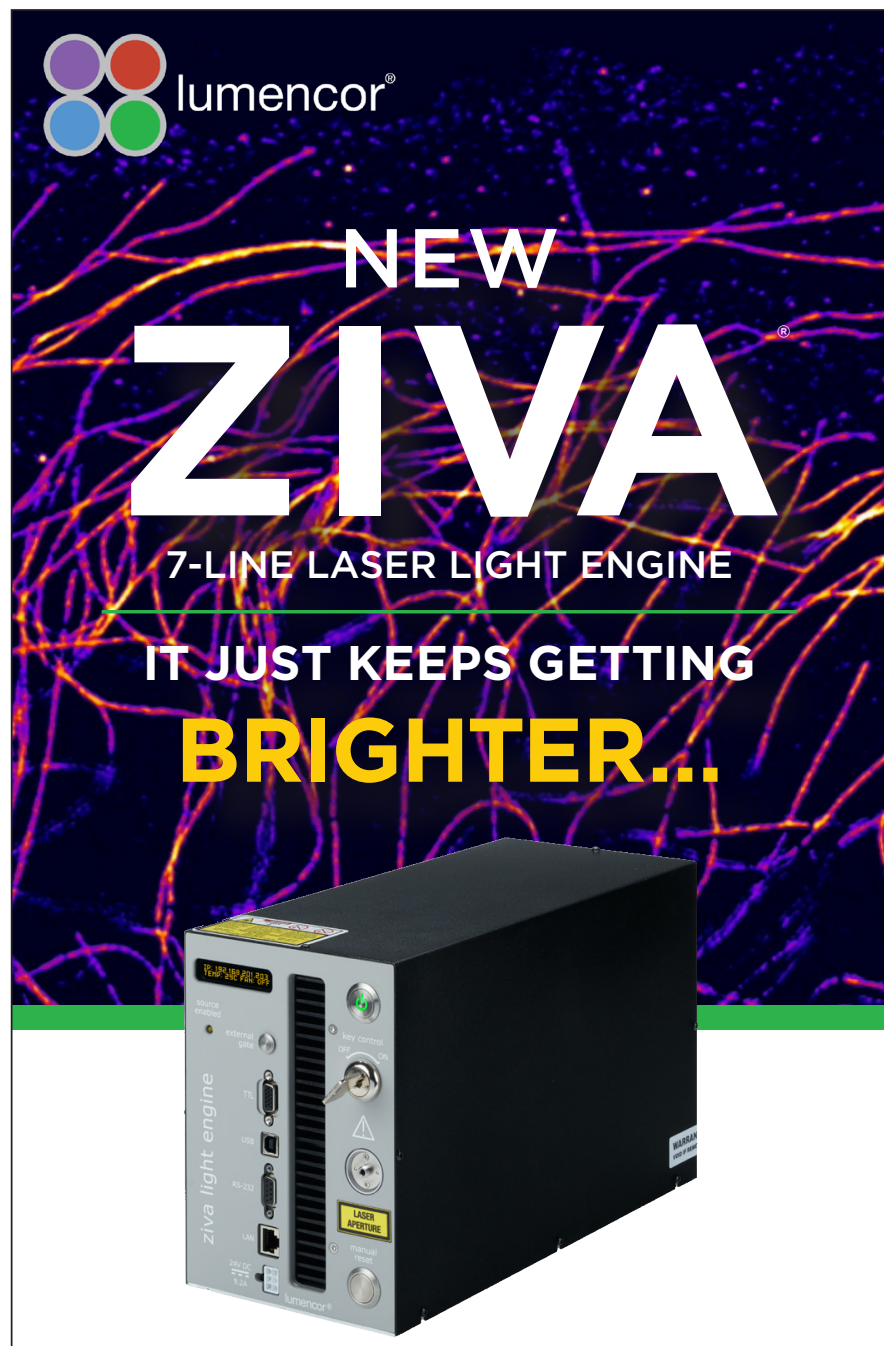

- Spectral breadth: $405,446,477,520$, 546, 638, 749nm

- Power: $100 \mathrm{~mW} /$ colorband at distal end of $100 \mu \mathrm{m}$ diameter fiber

- Control: Active power stabilization

- Stability: Exceptional reproducibility, ideal for quantitation

- Ease of use: Pre-aligned, independent lasers

- Applications: Confocal, SIM, super-resolution microscopy, optogenetics and more

- Off-the-shelf and custom configurations upon request

www.lumencor.com 